LIFTED POLYTOPE METHODS FOR COMPUTING THE JOINT
SPECTRAL RADIUS*

RAPHAËL M. JUNGERS ${ }^{\dagger}$, ANTONIO CICONE , AND NICOLA GUGLIELMI $^{\S}$

Abstract. We present new methods for computing the joint spectral radius of finite sets of matrices. The methods build on two ideas that previously appeared in the literature: the polytope norm iterative construction, and the lifting procedure. Moreover, the combination of these two ideas allows us to introduce a pruning algorithm which can importantly reduce the computational burden. We prove several theoretical properties of our methods, such as finiteness computational result which extends a known result for unlifted sets of matrices, and provide numerical examples of their good behavior.

Key words. joint spectral radius, lifting methods, iterative methods, matrix semigroups, semidefinite programming, finiteness property

AMS subject classifications. 93C $30,37 \mathrm{C} 75,90 \mathrm{C} 22,93 \mathrm{D} 30$

DOI. $10.1137 / 130907811$

1. Introduction. Many mathematical and real-life problems lead to the study of the stability of a discrete time linear switched system (LSS)

$$
x(k)=A_{i_{k}} x(k-1), \quad k=1,2, \ldots,
$$

where $x(0) \in \mathbb{C}^{n}$ and $A_{i_{k}} \in \mathbb{C}^{n \times n}$ are elements of a given bounded family of matrices

$$
\mathcal{M}=\left\{A_{i}\right\}_{i \in \mathcal{I}},
$$

and $\mathcal{I}$ is a set of indices. For recent surveys on stability criteria for switched (discrete and continuous) linear systems, we refer the reader to [19,24,32].

In particular, it is important to find necessary and sufficient conditions on the matrix family $\mathcal{M}$ ensuring that the LSS (1.1) is uniformly asymptotically stable in the following sense.

DEFINITION 1.1. The LSS (1.1) is said to be uniformly asymptotically stable if it holds that

$$
\lim _{k \rightarrow \infty} x(k)=0
$$

for any initial condition $x(0) \in \mathbb{C}^{n}$ and for any possible sequence of matrices $\left\{A_{i_{k}}\right\}_{k \geq 1}$ chosen in the family $\mathcal{M}$.

* Received by the editors January 29, 2013; accepted for publication (in revised form) by J. G. Nagy January 15, 2014; published electronically DATE. The authors were partially supported by the Italian GNCS under the Programma Giovani Ricercatori 2011.

http://www.siam.org/journals/simax/x-x/90781.html

†ICTEAM Institute, Université Catholique de Louvain, B-1348 Louvain-la-Neuve, Belgium (raphael.jungers@uclouvain.be). This author is an F.R.S.-FNRS Research Associate. His work was also supported by the communauté francaise de Belgique - Actions de Recherche Concertées and by the Belgian Programme on Interuniversity Attraction Poles initiated by the Belgian Federal Science Policy Office.

${ }^{\ddagger}$ School of Mathematics, Georgia Institute of Technology, Atlanta, GA 30332 (antonio.cicone@ univaq.it).

$\S$ Dipartimento di Ingegneria Scienze dell'Informazione e Matematica, Università de L'Aquila, 67100 L'Aquila, Italy (guglielm@univaq.it). 
As is well known, the LSS (1.1) is uniformly asymptotically stable if and only if the so-called joint spectral radius, or simply spectral radius, of the associated matrix family $\mathcal{M}=\left\{A_{i}\right\}_{i \in \mathcal{I}}$ is smaller than one [19]. We now properly define this notion.

For each $k=1,2, \ldots$, consider the set $\mathcal{P}_{k}(\mathcal{M})$ of all possible products of length $k$ whose factors are elements of $\mathcal{M}$; that is, $\mathcal{P}_{k}(\mathcal{M})=\left\{A_{i_{1}} \ldots A_{i_{k}} \mid i_{1}, \ldots, i_{k} \in \mathcal{I}\right\}$, and set

$$
\mathcal{P}(\mathcal{M})=\bigcup_{k \geq 1} \mathcal{P}_{k}(\mathcal{M})
$$

to be the multiplicative semigroup associated with $\mathcal{M}$.

We recall two possible generalizations of the concept of spectral radius of a single matrix to the case of a family of matrices $\mathcal{M}$.

Definition 1.2 (joint spectral radius [31]). If $\|\cdot\|$ is any matrix norm on $\mathbb{C}^{n \times n}$, consider $\widehat{\rho}_{k}(\mathcal{M}):=\sup _{P \in \mathcal{P}_{k}(\mathcal{M})}\|P\|^{1 / k}, k \in \mathbb{N}$, and define the joint spectral radius (jsr) of $\mathcal{M}$ as

$$
\widehat{\rho}(\mathcal{M})=\lim _{k \rightarrow \infty} \widehat{\rho}_{k}(\mathcal{M}) .
$$

The jsr does not depend on the matrix norm chosen thanks to the equivalence between matrix norms in finite dimensional spaces.

Definition 1.3 (generalized spectral radius [10]). Let $\bar{\rho}(\cdot)$ denote the spectral radius of an $n \times n$-matrix, consider $\bar{\rho}_{k}(\mathcal{M}):=\sup _{P \in \mathcal{P}_{k}(\mathcal{M})} \rho(P)^{1 / k}, k \in \mathbb{N}$, and define the generalized spectral radius (gsr) of $\mathcal{M}$ as

$$
\bar{\rho}(\mathcal{M})=\limsup _{k \rightarrow \infty} \bar{\rho}_{k}(\mathcal{M}) .
$$

In the case of bounded sets $\mathcal{M}$ Daubechies and Lagarias conjectured the equality of gsr and jsr, which was then proven by Berger and Wang [3]. From now on we shall restrict ourselves to finite sets of matrices; that is, we assume $\mathcal{I}$ is finite and we call the $j s r$ of a finite set $\mathcal{M}$ the quantity

$$
\rho(\mathcal{M}) \triangleq \widehat{\rho}(\mathcal{M})=\bar{\rho}(\mathcal{M})
$$

It is not difficult to see that for any bounded set of matrices

$$
\forall k, \bar{\rho}_{k}(\mathcal{M}) \leq \rho(\mathcal{M}) \leq \widehat{\rho}_{k}(\mathcal{M})
$$

A further important characterization of the jsr is the following.

Proposition 1.4 (see $[11,31]$ ). The spectral radius of a bounded family $\mathcal{M}$ of complex $n \times n$-matrices is characterized by the equality

$$
\rho(\mathcal{M})=\inf _{\|\cdot\| \in \mathrm{Op}}\|\mathcal{M}\|
$$

where $\mathrm{Op}$ is the set of operator norms and

$$
\|\mathcal{M}\|=\sup _{i \in \mathcal{I}}\left\|A_{i}\right\|=\widehat{\rho}_{1}(\mathcal{M})
$$

The jsr has found a great number of applications in several fields of engineering, mathematics, and computer science. See [33] and [19] for diverse applications. It is notoriously very hard to compute. Recently, several different methods have been 
proposed to provide approximations of this quantity $[1,9,12,23,25]$. In particular, there has been growing interest in the study of the so-called extremal norms $[7,21$, $26,30,35]$. For sets of matrices with jsr equal to one, these are norms whose unit ball is invariant under the action of the matrices in the set. This confers a practical interest to the analysis of such sets: if one manages to generate a norm whose unit ball is an invariant set, then this directly allows bounding the jsr: $\rho \leq 1$. Methods based on the generation of an invariant polytope have been proposed in $[14,17,18]$. A great advantage of these methods is that they can stop in finite time, and provide an exact characterization of the jsr of the set of matrices. If, besides the knowledge of an invariant polytope, one knows a product whose spectral radius is equal to one, then (by (1.6)) one has a proof that the exact value of the jsr is one.

Along another line of research, methods have been proposed which base their analysis on the existence of a common invariant cone for the set of matrices, or which first transform the set of matrices so that the new matrices share an invariant cone (see, e.g., $[5,20,27,29])$. In this paper, we show how to combine these two approaches, and how this combination allows one to derive innovative efficient algorithms for computing the jsr. Our work not only provides new efficient algorithms that can outperform state-of-the-art methods available in the literature, but also provides an improvement in our theoretical understanding of the jsr, as we exhibit novel algebraic constructions which allow one in many cases to finitely describe and compute the jsr. In the next section, we present in more detail these two ideas that are the basis of our work.

2. Extremal norms and invariant cones. The definitions and results reported in this section may be found in [14]. In the rest of the paper, we restrict ourselves to the case $\rho(\mathcal{M})>0$ since it can be efficiently decided whether $\rho=0[19$, section 1.2.2.9]. Thus, we use the notation

$$
\widehat{\mathcal{M}}=\frac{\mathcal{M}}{\rho(\mathcal{M})} \quad \Longrightarrow \quad \rho(\widehat{\mathcal{M}})=1 .
$$

Given a family $\mathcal{M}$, an important question is whether the infimum in (1.7) is a minimum or not. In this respect, we have the following definition.

Definition 2.1 (extremal norm). We say that a norm $\|\cdot\|_{*}$ satisfying the condition

$$
\|\mathcal{M}\|_{*}=\rho(\mathcal{M})
$$

is extremal for the family $\mathcal{M}$.

Already for single matrices, in general extremal norms are not assured to exist. A necessary and sufficient condition for the existence of an extremal norm is that the family $\mathcal{M}$ is nondefective.

Definition 2.2 (see $[15,31])$. A family of complex $n \times n$-matrices $\mathcal{M}$ is said to be nondefective if the multiplicative semigroup $\mathcal{P}(\widehat{\mathcal{M}})$ is a bounded set of matrices.

By a further characterization of defective families in terms of reducibility (recall that a set is reducible if there exists a common nontrivial invariant subspace) it was proved in $[2,35]$ that for a compact irreducible set $\mathcal{M}$ of matrices there exists an extremal norm.

An extremal norm is a norm whose unit ball is invariant under the action of the matrices in the normalized set. This confers a practical interest to the analysis of such objects: if one manages to generate a norm whose unit ball is an invariant set, then this directly allows one to bound the jsr: $\rho(\mathcal{M}) \leq 1$. 
To this aim we give the following definition.

Definition 2.3 (trajectory). Given a bounded family $\mathcal{M}$ of complex $n \times n$ matrices and a vector $x \in \mathbb{C}^{n}$, we say that

$$
\mathcal{T}[\widehat{\mathcal{M}}, x]=\{x\} \bigcup\{P x \mid P \in \mathcal{P}(\widehat{\mathcal{M}})\}
$$

is the trajectory of $\mathcal{M}$ starting from $x$.

Recall [16] that, given $\mathcal{X} \subset \mathbb{C}^{n}$, the $\operatorname{absco}(\mathcal{X})$ is the set of all the finite absolutely convex linear combinations of vectors of $\mathcal{X}$; i.e., $x \in \operatorname{absco}(\mathcal{X})$ if and only if there exist $x_{1}, \ldots, x_{k} \in \mathcal{X}$ with $k \geq 1$ such that $x=\sum_{i=1}^{k} \lambda_{i} x_{i}$ with $\lambda_{i}{ }^{\prime} \mathrm{s} \in \mathbb{C}$ and $\sum_{i=1}^{k}\left|\lambda_{i}\right| \leq 1$.

TheOrem 2.4 (see [14,22]). Let $\mathcal{M}$ be a nondefective bounded family of complex $n \times n$-matrices and, given a vector $x \in \mathbb{C}^{n}$, assume that the set $\mathcal{T}[\widehat{\mathcal{M}}, x]$ spans $\mathbb{C}^{n}$. Then the closure of the absolutely convex hull of the trajectory,

$$
\mathcal{S}[\widehat{\mathcal{M}}, x]=\operatorname{clos}(\operatorname{absco}(\mathcal{T}[\widehat{\mathcal{M}}, x]))
$$

is the unit ball of an extremal norm for $\mathcal{M}$.

If the family $\mathcal{M}$ is real and has an invariant real cone $K$, then the above theorem has been extended in [13].

2.1. Finite computation of the jsr by polytope extremal norms. The recent methods proposed in $[13,14,17,18,28]$ are all based on the computation of a polytope extremal norm; that is, a norm whose unit ball is a balanced real (or complex) polytope.

This kind of construction is often possible when the family $\mathcal{M}$ is finite and has the so-called finiteness property, which we now define.

Definition 2.5 (spectrum-maximizing product and the finiteness property). If $\mathcal{M}$ is a bounded family of complex $n \times n$-matrices, any matrix $\bar{P} \in \mathcal{P}_{k^{*}}(\mathcal{M})$ satisfying

$$
\rho(\bar{P})^{1 / k^{*}}=\bar{\rho}_{k^{*}}(\mathcal{M})=\rho(\mathcal{M})
$$

for some $k^{*} \geq 1$ is called a spectrum-maximizing product (SMP) for $\mathcal{M}$.

If $\mathcal{M}$ admits an $S M P$, then we say that it has the finiteness property.

Under some suitable further assumption on the family and a special choice of the starting vector $x$ (see, e.g., [14] and section 5), it is possible to construct an extremal polytope norm by means of Theorem 2.4.

The trajectory, in fact, can be constructed recursively as

$$
\mathcal{T}^{(k+1)}[\widehat{\mathcal{M}}, x]=\widehat{\mathcal{M}} \mathcal{T}^{(k)}[\widehat{\mathcal{M}}, x] .
$$

with $\mathcal{T}^{(0)}[\widehat{\mathcal{M}}, x]=\{x\}$. As a consequence, if $\mathcal{T}[\widehat{\mathcal{M}}, x]$ spans the whole space and

$$
\mathcal{T}^{(k+1)}[\widehat{\mathcal{M}}, x] \subseteq \mathcal{S}^{(k)}[\widehat{\mathcal{M}}, x]
$$

then $\mathcal{S}^{(k)}[\widehat{\mathcal{M}}, x]$ provides an extremal polytope norm which allows for a finite computation of $\rho(\mathcal{M})$, where $\mathcal{S}^{(k)}[\widehat{\mathcal{M}}, x]$ is given by replacing $\mathcal{T}$ with $\mathcal{T}^{(k)}$ in $(2.3)$.

Clearly, in order for the procedure (see [17] for more details) to be successful, it is necessary that $\mathcal{M}$ be properly scaled by $\rho(\mathcal{M})$, which means that an SMP has been detected (the success of the procedure implies this a posteriori). 
2.2. Invariant cone and the semidefinite lifting. We recall that if $V$ is a finite dimensional real or complex vector space, a subset $K \subset V$ is said to be a cone if and only if $x, y \in K, \alpha, \beta \geq 0$ implies that $\alpha x+\beta y \in K$. A cone $K$ is said to be pointed if and only if $K \cap(-K)=\{0\}$. By cone we mean a convex, closed, pointed cone with nonempty interior. Any such cone defines a partial order in $\mathbb{C}^{n}$ : we write $x \succeq_{K} y\left(x \succ_{K} y\right)$ for $x-y \in K(x-y \in \operatorname{int} K)$ [6, section 2.4.1]. The cone $K$ is an invariant cone for the matrix $A$ if $A K \subset K$. In this case we write $A \succeq_{K} 0$. If $K$ is invariant for all matrices of some set $\mathcal{M}$, then it is said to be an invariant cone for that set.

Recently Blondel and Nesterov [5] have introduced a lifting technique to compute the jsr. We generalize this notion to complex matrices.

Definition 2.6. Given a vector $v \in \mathbb{C}^{n}$, its semidefinite lifting is defined as

$$
\widetilde{v}=v \cdot v^{*}
$$

where $v^{*}$ is the conjugate transpose of $v$.

Also, the semidefinite lifting of a complex matrix $A$ is the linear operator

$$
\widetilde{A}: \mathcal{H}^{n} \rightarrow \mathcal{H}^{n}, \quad X \mapsto A X A^{*},
$$

where $\mathcal{H}^{n}$ denotes the $n(n+1) / 2$ dimensional vector space of Hermitian matrices.

The linear operator $\widetilde{A}$ can easily be represented by a matrix acting on $\mathbb{C}^{n(n+1) / 2}$ (see [5] for details). It is well known (see, e.g., [4]) that the set of $n \times n$ Hermitian positive semidefinite matrices defines a pointed cone inside the vector space of Hermitian $n \times n$-matrices. Such a cone plays an important role in the following discussion.

DEFINITION 2.7. The cone of Hermitian positive semidefinite matrices is the set

$$
\mathcal{H}_{+}^{n}=\left\{H \in \mathbb{C}^{n \times n}: H^{*}=H \forall x \in \mathbb{C}^{n}, x^{*} H x \geq 0\right\}=\left\{H \in \mathbb{C}^{n \times n}: H \succeq 0\right\} .
$$

In what follows we shall simply denote $\mathcal{H}_{+}^{n}$ as $\mathcal{H}$.

It follows that if $X \in \mathcal{H}$, then trivially $A X A^{*} \in \mathcal{H}$. Therefore, the cone $\mathcal{H}$ is invariant under such a linear operator $\widetilde{A}$. Moreover, the following property is also obvious.

Proposition 2.8. Let $A$ be a complex matrix. For any complex vector $v$, we have

$$
\widetilde{A}(\widetilde{v})=\widetilde{A v} .
$$

We indicate by $\widetilde{\mathcal{M}}$ the lifted family. The following important result relates the spectral radii of a family of matrices and the corresponding lifted set, and it is a straightforward generalization of a theorem proved by Blondel and Nesterov.

TheOrem 2.9 (see [5, Theorem 4]). For any bounded set of complex $n \times n$ matrices $\mathcal{M}$ it holds that

$$
\rho(\widetilde{\mathcal{M}})=\rho(\mathcal{M})^{2} .
$$

Thus, if one wants to compute the jsr of $\mathcal{M}$, it is sufficient to compute it for the transformed set $\widetilde{\mathcal{M}}$.

Our goal is to exploit the invariant cone property of the lifted set in order to compute the jsr by determining an extremal norm for $\widetilde{\mathcal{M}}$. As we will see, it will be sufficient to find an invariant set inside the invariant cone. Indeed, it will define the unit ball of a norm on the cone, that is, a function which fulfills the classical properties 
of a vector norm when applied to vectors of that cone. Even though these functions can be extended to regular norms on the whole state space, the properties they satisfy on the cone might not hold on the full state space. Nevertheless, we will show that verifying these properties on the sole cone will be sufficient for our purposes.

A convenient way of obtaining such an invariant set is given in the following definition. It is reminiscent of the convex hull operation, but, besides being defined by a set of points, it also depends on the geometry of an underlying cone.

Definition 2.10 (conical convex hull and conitope). Given a cone $K$ and a (possibly infinite) set of vectors $U \subset K$, its conical convex hull is defined as

$$
\operatorname{coniconv}(U) \triangleq\left\{x \in K: \exists y \in \operatorname{conv}(U): x \preceq_{K} y\right\},
$$

where conv denotes the usual convex hull of a set of vectors.

We say that a set $S \subset K$ is a conitope if there is a finite set $U=\left\{u_{j}\right\}_{j=1}^{\ell}$ such that

$$
S=\operatorname{coniconv}(U)
$$

The following definition characterizes minimal sets of vertices defining a conitope.

Definition 2.11 (essential system). Let $K$ be a cone. Given a set $U \subset K$, we say that $W \subset U$ is an essential system of vertices of $\operatorname{coniconv}(U)$ if $\operatorname{coniconv}(W)=$ $\operatorname{coniconv}(U)$, and if for any $W^{\prime} \subset W, W^{\prime} \neq W, \operatorname{coniconv}\left(W^{\prime}\right) \neq \operatorname{coniconv}(W)$.

Conitopes can, in turn, define a norm on the corresponding cone as follows.

Definition 2.12. Given a set of vectors $U$ inside a cone $K$ such that $\operatorname{conv}(U) \cap$ $\operatorname{int} K \neq \emptyset$, one can define for $x \in K$

$$
|x|_{U} \triangleq \inf \{\lambda>0: x \in \lambda \cdot \operatorname{coniconv}(U)\} .
$$

Lemma 2.13 (conitope norm). Let $K$ be a cone, and let $U$ be a set of points in $K$, such that $\operatorname{conv}(U) \cap \operatorname{int} K \neq \emptyset$. Then, the function $|\cdot|_{U}$ defined on $K$ satisfies the properties of a norm.

We call such functions conitope norms when coniconv $(U)$ has a finite essential system of vertices.

The idea in the lemma above is equivalent to the concept of Minkowski norm, except that we restrict our attention to vectors in the invariant cone (that is, the function obtained verifies all the requirements of a norm, when applied to vectors in the cone). The proof of the lemma is just standard computation, and goes by the same way as for actual Minkowski norms. We remark that similar ideas have appeared in the recent literature. If we restrict ourselves [13] to a set of real matrices $\mathcal{M}$ which does not have any common invariant face of a cone $K \subset \mathbb{R}^{n}, \mathcal{M}$ has a monotone (that is, $|x|>|y|$ if $x>_{K} y$ ) extremal norm on $K$ whose unit ball is obtained as

$$
\mathcal{S}[\widehat{\mathcal{M}}, x]=\left\{z \in K \text { such that } \exists y \in \operatorname{conv}(\mathcal{T}[\widehat{\mathcal{M}}, x]): z \preceq_{K} y\right\} .
$$

See also [30] for a similar existence theorem with $K=\mathbb{R}_{+}^{n}$.

We finally note that thanks to modern optimization techniques like interior point methods, the computation of both coniconv $(U)$ and $|x|_{U}$ can be performed in polynomial time in case $K=\mathbb{R}_{+}^{n}$ (the positive orthant), $K=\mathcal{S}$ (the set of real symmetric positive definite matrices), or $K=\mathcal{H}$. See [29] and [6] for explicit formulations of these problems as linear programs (resp., LMIs). As the reader will see, our methods heavily rely on subroutines for performing these computations. 
3. The novel methods. We now show how to combine the two approaches described in sections 2.1 and 2.2. Our goal is to find conitope norms which are extremal for a given set of matrices. We will show that this combination allows one to derive innovative efficient algorithms for computing the jsr. In this section, we present the algorithms that unify the two approaches. Then, in sections 4 and 5 we provide the main theoretical results. Finally, in section 6 we give evidence for the good performance of our methods on numerical examples.

3.1. Two algorithms. We present here two algorithms. They both generate an upper and a lower bound which converge towards the jsr. We denote as L.E.(A) a leading eigenvector of a matrix $A$.

Algorithm 1: The conitope method. We describe in Algorithm 1 the basic method to compute an invariant conitope. Its main idea is to iteratively build an invariant conitope by taking the successive images of a starting vector, and testing at every step whether the new generated vectors belong to the conitope generated by the previous ones. If an invariant set is reached, then it provides a proof that $\rho(\mathcal{M} / C) \leq 1$.

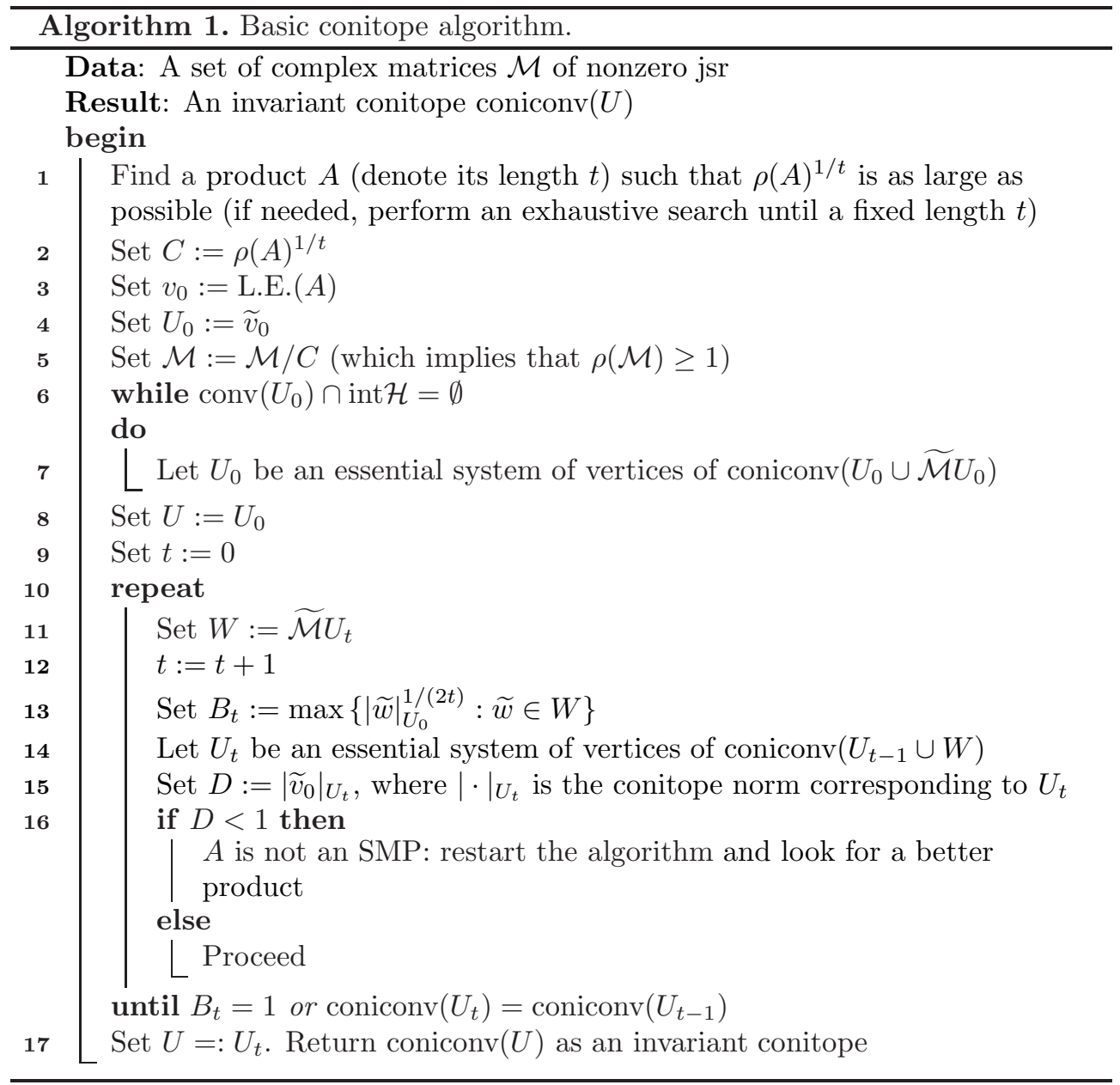


Algorithm 2: The dynamical procedure. In Algorithm 2 we describe a modification of Algorithm 1 which is allowed by the fact that the matrices share a common invariant cone. We exploit more the fact that the cone induces a partial order relation between points, which allows us to discard some of them, if they are dominated by another point.

This algorithm is an iterative version of the previous one: while trying to prove that a certain product $A$ is an SMP, at the same time it tries to find another, better product $A^{\prime}$. Once it finds such a product, the algorithm updates the lower bound and simply continues its computation (note that one can rescale the vertices in $U_{t}$ by a common factor if they grow too much; this does not change the outcome of the algorithm as the system is homogeneous).

Line 12 in the algorithm deserves an explanation: there, we update the lower bound by computing the spectral radius of some products (averaged by their length). We manage to prove below that one only has to look at a restricted set of products in order to have a lower bound that converges towards the actual jsr: these are products that generate the vertices of the conitope, as well as their subproducts. We direct the reader to the fact that by subproducts, we mean all the consecutive matrix products in a particular product (for instance, $A_{2} A_{2}$ is a subproduct of $A_{1} A_{2} A_{2} A_{1}$, but $A_{1} A_{2} A_{1}$ is not).

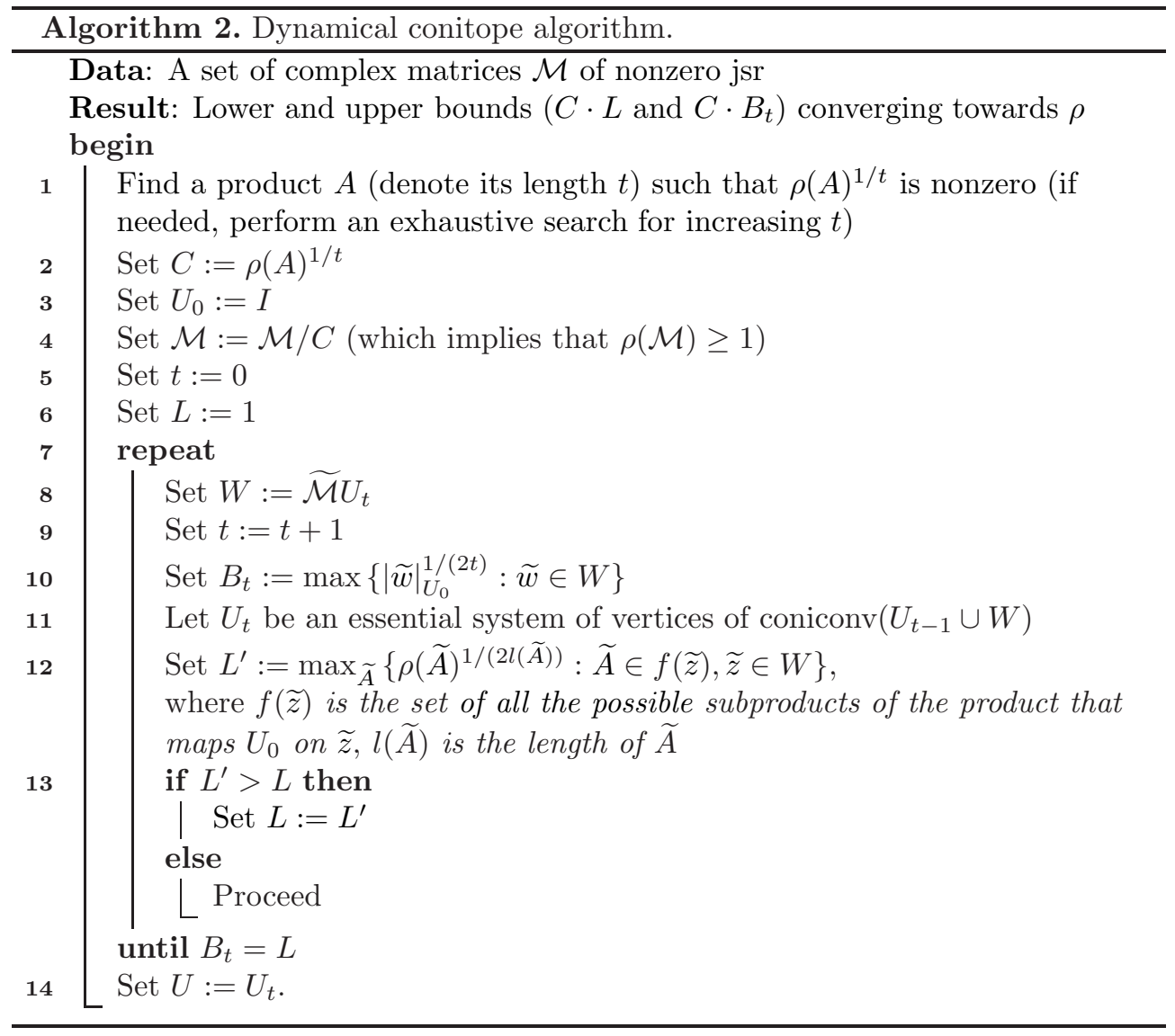


Remark 1. The algorithms differ by two facts: first, in the initialization part, the first one starts with a leading eigenvector of a candidate SMP (at the cost of recomputing longer and longer products until it finds a better candidate SMP every time it goes back to line 1), while the second one starts with the identity matrix; second, in the first algorithm, one reinitializes the conitope, by restarting from the new leading eigenvector, while the second algorithm keeps all the previous vertices and continues with them. These two modifications are independent from each other, but the main idea is that in the first algorithm, starting with a leading eigenvector is a necessary condition in order to stop in finite time [14, small CPE theorem]. For the second algorithm, we start with the identity matrix because we do not really hope to converge in finite time, but we are more interested in obtaining rapidly converging bounds than having a finite time termination. Note, finally, that the choice of the identity matrix is arbitrary and one could choose any other starting point in the interior of the cone.

Finally, in Algorithm 1 we do not search for a better spectral radius among all the subproducts, like in Algorithm 2. However, one could perform this search as well in Algorithm 1, which would allow one to potentially find a better SMP without having to resort to an exhaustive search when coming back to the preprocessing phase.

4. Bounds and convergence results. In this section, we prove that our methods effectively provide lower and upper bounds on the jsr, and that, moreover, these bounds converge towards the exact value asymptotically.

Proposition 4.1. Let $\widetilde{\mathcal{M}}$ be a set of lifted matrices that share an invariant cone $K$. Let $U$ be a set of points in $K$, such that $\operatorname{conv} U \cap \operatorname{int} K \neq \emptyset$. Then, we have the following upper bound on the jsr:

$$
\rho(\widetilde{\mathcal{M}}) \leq \widehat{\rho}_{U}(\widetilde{\mathcal{M}}):=\sup _{\widetilde{A} \in \widetilde{\mathcal{M}}} \sup _{\widetilde{u} \in U}|\widetilde{A} \widetilde{u}|_{U}
$$

Proof. By (1.5), for any $\epsilon>0$, there exists a $t$ and a product $\widetilde{A} \in \mathcal{P}_{t}(\widetilde{\mathcal{M}})$ such that $\rho(\widetilde{A}) \geq(\rho(\widetilde{\mathcal{M}})-\epsilon)^{t}$. Since the matrices in $\widetilde{\mathcal{M}}$ share an invariant cone $K$, by the generalized Perron-Frobenius theorem (see, for instance, [20, Theorem 1]), there exists a vector $\widetilde{u} \in K$ such that $\widetilde{A} \widetilde{u}=\left(\rho(\widetilde{\mathcal{M}})-\epsilon^{\prime}\right)^{t} \widetilde{u}$ for some $0<\epsilon^{\prime}<\epsilon$. Let us take such a vector $\widetilde{u}$ with $|\widetilde{u}|_{U}=1$. Now, $\widetilde{A} \widetilde{u} \in \operatorname{coniconv}\left(\widehat{\rho}_{U}(\widetilde{\mathcal{M}})^{t} U\right)$ (as implied by (4.1)) together with $\widetilde{A} \widetilde{u}=\left(\rho-\epsilon^{\prime}\right)^{t} \widetilde{u}$ imply

$$
\widehat{\rho}_{U}(\widetilde{\mathcal{M}}) \geq \rho-\epsilon^{\prime} .
$$

Since this must hold for any $\epsilon$ we obtain (4.1).

Since Algorithm 1 starts with a set of vectors $U_{0}$, which are rank one matrices, the set $U_{t}$ actually contains only rank one matrices during the whole run of the algorithm. Hence, it is not clear that one obtains for sure a norm (as defined by Lemma 2.13). The next proposition shows how to deal with this problem.

Proposition 4.2. If the $n \times n$-matrices in $\mathcal{M}$ do not have a nontrivial common invariant complex subspace, then Algorithm 1 generates in finite time a set $U$ such that $\operatorname{conv}(U) \cap \operatorname{int} \mathcal{H} \neq \emptyset$.

Proof. Recall that we start with $v_{0}$ a leading eigenvector of $A$. If $\mathcal{M}$ does not have any invariant subspace, we can find $A_{1}, \ldots, A_{n}$, which are products of matrices in $\mathcal{M}$ of length at most $n$, such that $\left\{A_{i} v_{0}\right\}_{i=1}^{n}$ spans $\mathbb{C}^{n}$. Now, it is direct to prove 
that $Q=\frac{1}{n} \sum_{i=1}^{n} A_{i} v_{0} v_{0}^{*} A_{i}^{*} \in \operatorname{conv}(U)$ at step $n$, and the matrix $Q$ has full rank; this proves the result.

Remark 2. We observe that if the algorithm does not generate such a set $U$ after $n$ iterations, then, given $v_{0}$ leading eigenvector of the matrix $A$ defined in Algorithm $1, S=\operatorname{span}\left(\mathcal{P}(\mathcal{M}) v_{0}\right)$ is an invariant complex subspace, and one can project the matrices on $S$ and on $S^{\perp}$ and iterate the algorithm.

In the proof of our main theorem below, we also need the following technical proposition.

Proposition 4.3 (see [11]). Let $\|\cdot\|$ be a matrix norm in $\mathbb{C}^{n}$ induced by the vector norm $|\cdot|$. There exists a constant $C(n)>0$ such that for all $z \in \mathbb{C}^{n},|z|=1$, and all $A \in \mathbb{C}^{n \times n},\|A\| \leq 1$, there is an eigenvalue $\lambda$ of $A$ such that

$$
|1-\lambda| \leq C|A z-z|^{1 / n}
$$

THEOREM 4.4. If the matrices in $\mathcal{M}$ do not have a nontrivial common invariant complex subspace, both Algorithms 1 and 2 provide an upper bound $\left(C B_{t}\right)$ and a lower bound (resp., $C$ and $C L$ ) which asymptotically converge towards the jsr.

Proof. In the following, $\rho$ denotes $\rho(\widetilde{\mathcal{M}})=\rho(\mathcal{M})^{2}$. We also call $\mathcal{M}^{\prime}$ the scaled set of matrices: $\mathcal{M}^{\prime}=\mathcal{M} / C$.

Lower bound. We want to prove that the lower bound converges towards the exact value of the jsr. Thus, for Algorithm 1, it suffices to show that if $\rho\left(\mathcal{M}^{\prime}\right)>1$ (i.e., if $A$ is not an SMP), the main loop terminates in finite time, and the algorithm will hence look for longer products by returning to the preprocessing phase. Since we have the well-known alternative definition (1.5) for the jsr, the lower bound will converge towards $\rho$.

Let us take a product $A \in \mathcal{P}\left(\mathcal{M}^{\prime}\right)$ such that $\rho(A)>1$ and $v$ its eigenvector: $A v=$ $r v, r>1$. By the same reasoning as in Proposition 4.2, we can suppose that we have $n$ linearly independent vectors $u_{j}=A_{j} v_{0}$, and $n$ others $v_{i}=A_{i} v, A_{i}, A_{j} \in \mathcal{P}\left(\mathcal{M}^{\prime}\right)$, $1 \leq i, j \leq n$. (Recall that $v_{0}$ is the initial starting point in Algorithm 1.) Thus, there exist $\lambda_{1}$ such that $\lambda_{1} \widetilde{v}_{0} \preceq \sum \widetilde{v}_{i} / n$, and $\lambda_{2}$ such that $\lambda_{2} \widetilde{v} \preceq \sum \widetilde{u}_{i} / n$. Now, taking $k$ large enough so that $r^{k} \lambda_{2} \lambda_{1}>1$, we have

$$
\widetilde{v}_{0} \preceq \sum \widetilde{v}_{i} /\left(n \lambda_{1}\right) \prec r^{k} \lambda_{2} \sum \widetilde{v}_{i} / n \preceq \lambda_{2} \sum \widetilde{A}_{i} \widetilde{A}^{k} \widetilde{v} / n \preceq \sum \widetilde{A}_{i} \widetilde{A}^{k}\left(\sum \widetilde{A}_{j} \widetilde{v}_{0}\right) / n^{2} .
$$

Hence, the algorithm will terminate because the last equation implies that $\widetilde{v}_{0}$ finally lies inside coniconv $\left(U_{t}\right)$ at some time, which is exactly the termination condition in Algorithm 1.

For Algorithm 2, recall from Proposition 4.1 that for any $t \geq 0$ and any norm $|\cdot|$,

$$
\rho(\widetilde{\mathcal{M}})^{t} \leq \sup _{\widetilde{A} \in \mathcal{P}_{t}(\widetilde{\mathcal{M}})} \sup _{\widetilde{u}:|\widetilde{u}|=1}|\widetilde{A} \widetilde{u}|
$$

Now, it is easy to show by induction that at every time $t$, every point of the type $\widetilde{A}_{t} \ldots \widetilde{A}_{1} \widetilde{u}_{0}$ belongs to coniconv $\left(U_{t}\right)$ (where $\widetilde{u}_{0}$ is a point in $U_{0}$ ). This is due to the fact that if $\widetilde{v} \preceq \sum \lambda_{i} \widetilde{u}_{i}$, then $\widetilde{A} \widetilde{v} \preceq \sum \lambda_{i} \widetilde{A} \widetilde{u}_{i}$.

For each time $t$, there is a point $\widetilde{v} \in \mathcal{P}_{t}(\widetilde{\mathcal{M}}) \widetilde{v}_{0}, \widetilde{v} \preceq \sum \lambda_{i} \widetilde{u}_{i}: \widetilde{u}_{i} \in U_{t}$ whose norm is larger than $\rho^{t}$. We can suppose that our norm is monotone, and thus $|v| \leq\left|\sum \lambda_{i} u_{i}\right|$. By convexity of the norm, it implies that there is at least one vertex $\widetilde{u}_{i} \in U_{t}$ with this 
property. That is, at each time $t$ there is a vertex $\widetilde{u} \in U$ whose norm is larger than $\rho^{t}$. We conclude that there exists an infinite product ... $\widetilde{A}_{t} \ldots \widetilde{A}_{1}$ such that $\widetilde{A}_{t} \ldots \widetilde{A}_{1} \widetilde{u}_{0}$ is in $U_{t}$ at time $t$, and a sequence $t_{1}, t_{2}, \ldots$ such that $\forall i,\left|\widetilde{A}_{t_{i}} \ldots \widetilde{A}_{1} U_{0}\right| \geq \rho^{t_{i}}$. (This latter step is a rather classical application of Konig's infinity lemma (see, e.g., [11, Lemma 3]).)

The end of the proof follows the same line of reasoning as in the main theorem of [11]. Let us now consider the normalized sequence $\widetilde{u}_{t_{i}}^{\prime}=\widetilde{A}_{t_{i}} \ldots \widetilde{A}_{1} U_{0} /\left|\widetilde{A}_{t_{i}} \ldots \widetilde{A}_{1} U_{0}\right|$. This is an infinite sequence on the boundary of the unit ball of the norm. By compactness of the boundary, there exists a subsequence $s_{i}$ such that the $\widetilde{u}_{s_{i}}^{\prime}$ converge to a vector $\widetilde{y}:|\widetilde{y}|=1$. So for any $\epsilon$, there must exist $s_{j}$ such that for all $s_{i}>s_{j}$,

$$
\left|\widetilde{u}_{s_{j}}^{\prime}-\widetilde{u}_{s_{i}}^{\prime}\right|<\epsilon .
$$

Setting $\widetilde{z}=\widetilde{u}_{s_{j}}^{\prime}, \widetilde{B}_{i}=\widetilde{A}_{s_{i}} \ldots \widetilde{A}_{s_{j}+1}\left(\left|\widetilde{u}_{s_{j}}\right| /\left|\widetilde{u}_{s_{i}}\right|\right)$, we get matrices $\widetilde{B}_{i}$ such that

$$
\left|\widetilde{B}_{i} \widetilde{z}-\widetilde{z}\right|<\epsilon,
$$

and we can conclude by Proposition 4.3 that $\widetilde{B}_{i}$ has an eigenvalue $\lambda$ such that $|1-\lambda|=$ $O\left(\epsilon^{1 / n}\right)$. Thus, since $\left(\left|\widetilde{u}_{s_{i}}\right| /\left|\widetilde{u}_{s_{j}}\right|\right)^{1 /\left(s_{i}-s_{j}\right)}$ tends to $\rho$,

$$
\rho\left(\widetilde{A}_{s_{i}} \ldots \widetilde{A}_{s_{j}+1}\right)^{1 /\left(s_{i}-s_{j}\right)} \rightarrow \rho .
$$

As a conclusion, Algorithm 2, which checks the spectral radius of all the products of the type $\widetilde{A}_{t_{i}} \ldots \widetilde{A}_{t_{j}}$ (that is, all the subproducts of the products corresponding to vertices of the conitope), will find a candidate SMP whose averaged spectral radius tends to $\rho$.

Upper bound. Recall that for any norm on the cone, the right-hand side of (4.1) (to the power $1 / t$ ) forms a sequence of approximations for $\rho$ :

$$
F_{t}=\sup _{\widetilde{A} \in \mathcal{P}_{t}(\widetilde{\mathcal{M}})}\left(\sup _{\widetilde{u}:|\widetilde{u}|=1}|\widetilde{A} \widetilde{u}|^{1 / t}\right) .
$$

From Definition 1.2 and (4.1) it follows that $\rho(\widetilde{\mathcal{M}}) \leq F_{t} \leq \widehat{\rho}_{t}(\widetilde{\mathcal{M}})$; hence $F_{t}$ tends to $\rho$ as $t \rightarrow \infty$. Also, we have shown above that at time $t$, for any $\widetilde{A} \in \mathcal{P}_{t}\left(\widetilde{\mathcal{M}}^{\prime}\right)$, and any $\widetilde{u} \in U_{0}, \widetilde{A} \widetilde{u} \in \operatorname{coniconv}\left(U_{t}\right)$.

This implies by convexity of the norm that

$$
\rho\left(\widetilde{\left(\mathcal{M}^{\prime}\right)}\right)^{t} \leq \max _{\widetilde{w} \in U_{t}}|\widetilde{w}|_{U_{0}}:=B_{t}^{2 t} .
$$

Thus, we have a sequence of upper bounds $Y_{t}=C^{2} B_{t}^{2}$ on $\rho$. Observe that

$$
Y_{t} \leq \max _{t^{\prime} \leq t} F_{t^{\prime}}^{\left(t^{\prime} / t\right)}
$$

This is because the vertices of $U_{t}$ are part of the images of vectors in $U_{0}$ (under products of diverse lengths $t^{\prime} \leq t$ ). It is straightforward to show that since $F_{t} \rightarrow \rho=$ $\rho(\mathcal{M})^{2},(4.4)$ implies that so does $Y_{t}=C^{2} B_{t}^{2}$.

Remark 3. If the set $\mathcal{M}$ is not irreducible, i.e., if there exists a nontrivial common invariant subspace, then we can still use the algorithms, and Theorem 4.4 remains valid, except for Algorithm 1, if we were not able to generate a full dimensional set $U_{0}$. In this case, we can detect the invariant subspace by Proposition 4.2, and apply the algorithm on the sets of smaller matrices. 


\section{Finiteness results.}

5.1. Preliminary results. We recall that $\widehat{\mathcal{M}}$ is the normalized family $\frac{\mathcal{M}}{\rho(\mathcal{M})}$.

Now we report the existence results proved in [14], which involve the normalized trajectories (2.2). First we observe that all the cyclic permutations of a product $\bar{P}$ have the same eigenvalues with the same multiplicities. Thus, if $\bar{P}=A_{i_{k^{\prime}}} \ldots A_{i_{1}}$ is an SMP for a family $\mathcal{M}$, then each of its cyclic permutations

$$
A_{i_{s}} \ldots A_{i_{1}} A_{i_{k^{\prime}}} \ldots A_{i_{s+1}}, \quad s=1, \ldots, k^{\prime}-1,
$$

are still an SMP for $\mathcal{M}$.

DeFinition 5.1 (asymptotically simple set). A nondefective bounded family $\mathcal{M}$ of complex $n \times n$-matrices is called asymptotically simple if it has a minimal SMP $\bar{P}$ with only one leading eigenvector (modulo scalar nonzero factors) such that the set $X$ of the leading eigenvectors of $\mathcal{M}$ is equal to the set of the leading eigenvectors of $\bar{P}$ and of its cyclic permutations.

We remark that the asymptotic simplicity of $\mathcal{M}$ means that the set $X$ of the leading eigenvectors of $\mathcal{M}$ is $\mathcal{M}$-cyclic, i.e., for any pair $(x, y) \in X \times X$ there exist $\alpha, \beta \in \mathbb{C}$ with

$$
|\alpha| \cdot|\beta|=1
$$

and two normalized products $\widehat{P}, \widehat{Q} \in \mathcal{P}(\widehat{\mathcal{M}})$ such that

$$
y=\alpha \widehat{P} x \quad \text { and } \quad x=\beta \widehat{Q} y .
$$

Theorem 5.2 (see [14]). Assume that a finite family $\mathcal{M}$ of complex $n \times n$ matrices is nondefective and asymptotically simple. Moreover, let $x \neq 0$ be a leading eigenvector of $\mathcal{M}$ and assume that $\operatorname{span}(\mathcal{T}[\widehat{\mathcal{M}}, x])=\mathbb{C}^{n}$. Then the set

$$
\partial \mathcal{S}[\widehat{\mathcal{M}}, x] \bigcap \mathcal{T}[\widehat{\mathcal{M}}, x]
$$

is finite (modulo scalar factors of unitary modulus). As a consequence, there exist a finite number of normalized products $\widehat{P}_{1}, \ldots, \widehat{P}_{s} \in \mathcal{P}(\widehat{\mathcal{M}})$ such that

$$
\mathcal{S}[\widehat{\mathcal{M}}, x]=\operatorname{absco}\left(\left\{x, \widehat{P}_{1} x, \ldots, \widehat{P}_{s} x\right\}\right),
$$

so that $\mathcal{S}[\widehat{\mathcal{M}}, x]$ is an invariant balanced complex polytope [16].

5.2. Asymptotic simplicity and extremal conitopes. In the following, by $\operatorname{conv}(U)$ we mean the convex hull of the adherence, that is, the closure points, of $U$.

Lemma 5.3. If a sequence of vectors $s_{i} \in \mathbb{C}^{n}$ converges to zero, then the set $\operatorname{coniconv}\left(\left\{\widetilde{s}_{i}\right\}\right)$ is a conitope.

Proof. Define $W=\operatorname{span}\left(\left\{s_{i}\right\}_{i>0}\right), k=\operatorname{dim}(W)$, and take a set $\left\{r_{1}, \ldots, r_{k}\right\} \subset$ $\left\{s_{i}\right\}_{i>0}$ of full rank. Then, all the $s_{i}$ can be written as $\sum_{j=1}^{k} \lambda_{j} r_{j}$.

Now take a vector $s_{i}$ with $i$ large enough such that

$$
\left\{r_{1}, \ldots, r_{k}\right\} \subset\left\{s_{1}, \ldots, s_{i-1}\right\},
$$


and such that, denoting $s_{i}=\sum_{j=1}^{k} \lambda_{j} r_{j},\left|\lambda_{j}\right|<1 / k^{2}$. We claim that

$$
s_{i} s_{i}^{*} \preceq_{\mathcal{H}} \sum_{j=1}^{k}\left(r_{j} r_{j}^{*}\right) / k .
$$

Indeed, taking an arbitrary vector $x \in \mathbb{C}^{n}$, we have

$$
\begin{aligned}
x^{*} s_{i} s_{i}^{*} x & =\sum_{j_{1}, j_{2}=1}^{k}\left(\lambda_{j_{1}} \lambda_{j_{2}}\right)\left(x^{*} r_{j_{1}} r_{j_{2}}^{*} x\right) \leq \sum_{j_{1}, j_{2}=1}^{k}\left|\lambda_{j_{1}}\right|\left|\lambda_{j_{2}}\left\|r_{j_{1}}^{*} x\right\| r_{j_{2}}^{*} x\right| \\
& \leq k^{2}\left(1 / k^{2}\right)^{2} \max _{j}\left|r_{j}^{*} x\right|^{2} \leq x^{*}\left(\sum_{j=1}^{k}\left(r_{j} r_{j}^{*}\right) / k\right) x .
\end{aligned}
$$

Since it is true for any $x \in \mathbb{C}^{n},(5.4)$ follows by Definition 2.7 .

As usual we indicate $\widetilde{s}=s \cdot s^{*}$. Equations (5.3) and (5.4) together imply that

$$
\widetilde{s}_{i} \in \operatorname{coniconv}\left(\left\{\widetilde{s}_{1}, \ldots, \widetilde{s}_{i-1}\right\}\right),
$$

and then,

$$
\operatorname{coniconv}\left(\left\{\widetilde{s}_{1}, \ldots, \widetilde{s}_{i}\right\}\right)=\operatorname{coniconv}\left(\left\{\widetilde{s}_{1}, \ldots, \widetilde{s}_{i-1}\right\}\right) .
$$

Now, take $i$ such that

$$
\forall l>i, \exists \lambda_{1}, \ldots, \lambda_{k}:\left|\lambda_{j}\right|<1 / k^{2}, \quad s_{l}=\sum_{j=1}^{k} \lambda_{j} r_{j}
$$

Thus, for $i$ large enough we have that for all $l>i$,

$$
\operatorname{coniconv}\left(\left\{\widetilde{s}_{1}, \ldots, \widetilde{s}_{l}\right\}\right)=\operatorname{coniconv}\left(\left\{\widetilde{s}_{1}, \ldots, \widetilde{s}_{i-1}\right\}\right),
$$

which implies that the limit of this set actually is a conitope, since the limit is reached for a finite number of vertices.

THEOREM 5.4. If a finite set of matrices $\mathcal{M}$ is irreducible and asymptotically simple, then its lifted set $\widetilde{\mathcal{M}}$ admits an invariant conitope. Moreover, there exists such an invariant conitope with vertices that are all rank one matrices.

Proof. The structure of the proof is analogous to that of Theorem 5.18 in [14].

We assume without loss of generality that $\rho(\mathcal{M})=\rho(\widetilde{\mathcal{M}})=1$. Let us denote by $X$ the finite set of leading eigenvectors (modulo scalar nonzero factors) of $\mathcal{M}$. Take a particular $v \in X$ and denote by $V$ the union of trajectories starting at $\widetilde{v}=v \cdot v^{*}$ under arbitrary products of matrices in $\widetilde{\mathcal{M}}$ :

$$
V=\left\{\widetilde{A}_{i_{t}} \ldots \widetilde{A}_{i_{1}} \widetilde{v}: \widetilde{A}_{i_{j}} \in \widetilde{\mathcal{M}} \forall j\right\} .
$$

We now define

$$
S=\operatorname{coniconv}(V) \text {. }
$$

Since $\widetilde{\mathcal{M}}$ is irreducible, again by [19, Theorem 2.1], the set $V$ is bounded, and $S$ is an invariant set for $\widetilde{\mathcal{M}}$. The last follows by the fact that if $U$ is an invariant set, then 
$\operatorname{coniconv}(U)$ is also an invariant set. We will prove that there exist a finite number of vectors $\widetilde{v}_{1}, \ldots, \widetilde{v}_{l} \in V$ such that $S=\operatorname{coniconv}\left(\left\{\widetilde{v}_{1}, \ldots, \widetilde{v}_{l}\right\}\right)$, and so $S$ is an invariant conitope for $\widetilde{\mathcal{M}}$.

Suppose the contrary. Then, there must be an infinite set of vectors $V^{\prime} \subset V$ such that

$$
\forall \widetilde{v}_{i} \in V^{\prime}, \widetilde{v}_{i} \notin \operatorname{coniconv}\left(V^{\prime} \backslash \widetilde{v}_{i}\right) .
$$

Moreover, by compactness of the set $S$ we can suppose that $V^{\prime}$ is a subset of a single trajectory of $\widetilde{v}$, that is, there exist indices $i_{1}, i_{2}, \ldots$ such that the set

$$
V^{\prime}=\left\{\widetilde{A}_{i_{t}} \ldots \widetilde{A}_{i_{1}} \widetilde{v}: t \geq 0\right\}
$$

contains an infinite set satisfying (5.6). (For a detailed proof of this fact, see, for instance, [14, Lemma 5.19].)

We note $\widetilde{v}_{t}=\widetilde{A}_{i_{t}} \ldots \widetilde{A}_{i_{1}} \widetilde{v}$, and correspondingly $v_{t}=A_{i_{t}} \ldots A_{i_{1}} v$. Since this set is infinite, there must be an index $t_{0}$ such that $v_{t_{0}} \in X, v_{t_{0}+1} \notin X$. Now, by compactness of $\operatorname{coniconv}\left(V^{\prime}\right)$, there must exist a subsequence of $\widetilde{v}_{t}$ that converges to a vector $\widetilde{w}$. This vector $\widetilde{w}$ is different from zero because, by (5.6) and Lemma 5.3, the sequence $\widetilde{A}_{i_{t}} \ldots \widetilde{A}_{i_{1}} \widetilde{v}$ is bounded away from zero. Then, since

$$
\widetilde{v}_{t}=v_{t} \cdot v_{t}^{*}
$$

this must be the case also for the original (unlifted) matrices: hence

$$
\exists t_{0}<t_{1}<t_{2}<\cdots t_{k} \cdots: A_{i_{t_{k}}} \ldots A_{i_{t_{0}+2}} v_{t_{0}+1} \rightarrow w
$$

and then $w \in X$. Now, by asymptotic simplicity, there exists a matrix $P \in \mathcal{P}(\mathcal{M})$ such that $P w=v_{t_{0}}$. This implies that $P A_{i_{t_{k}}} \ldots A_{i_{t_{0}+2}} v_{t_{0}+1} \rightarrow v_{t_{0}}$, and hence, premultiplying the above equation by $A_{i_{t_{0}+1}}$, we obtain that

$$
A_{i_{t_{0}+1}} P A_{i_{t_{k}}} \ldots A_{i_{t_{0}+2}} v_{t_{0}+1} \rightarrow v_{t_{0}+1}
$$

and $v_{t_{0}+1}$ is in $X$, which gives a contradiction.

Remark 4. Note that if one applies the polytope algorithm in $[14,18]$ and then lifts the vertices $\left\{v_{i}\right\}$ and considers the corresponding conitope, one does not obtain, in general, an invariant set for the lifted set of matrices. This is a key point in order to understand that the two algorithms are fundamentally different (see the following Example 3 in section 6).

\section{Examples and numerical tests.}

6.1. Illustrative examples. We provide here some illustrative examples showing advantages of the methods proposed in this paper.

Example 1. Consider the set of matrices

$$
\mathcal{M}=\left\{\left(\begin{array}{rrrr}
0 & -1 & 1 & 1 \\
1 & 0 & 0 & 0 \\
0 & -1 & 0 & 0 \\
1 & -1 & -1 & 0
\end{array}\right), \quad\left(\begin{array}{rrrr}
0 & -1 & 1 & 0 \\
-1 & -1 & 1 & 1 \\
-1 & 0 & 0 & 0 \\
-1 & -1 & 0 & -1
\end{array}\right)\right\}
$$

This set of matrices has a jsr

$$
\rho(\mathcal{M})=\rho\left(A_{2}\right) \approx 1.7779,
$$


where $A_{2}$ has two complex and conjugate leading eigenvalues. The classical balanced complex polytope (BCP) algorithm terminates in 4 steps and finds a $\mathrm{BCP}$ which is the convex hull of 16 vertices. Algorithm 1 terminates after two steps and finds an invariant conitope with only seven vertices.

Example 2. Our next example presents a set of complex matrices

$$
\begin{aligned}
& \mathcal{M}=\left\{\left(\begin{array}{rrr}
-1+i & -i & -1+i \\
0 & 1 & -1-i \\
1+i & -i & -1-i
\end{array}\right),\right. \\
& \left.\left(\begin{array}{rrr}
i & -1-i & -1 \\
1-i & -1+i & i \\
-1+i & 1+i & 1+i
\end{array}\right)\right\} .
\end{aligned}
$$

In this case $\rho(\mathcal{M})=\rho\left(A_{1}^{2} A_{2} A_{1} A_{2}\right) \approx 2.2401$.

The classical BCP algorithm requires 20 steps to find a BCP with 65 essential vertices. Algorithm 1 terminates after eight steps and the invariant conitope has only ten essential vertices.

Example 3. A set of matrices for which $\operatorname{conv}\left(\left\{v_{i}\right\}\right)$ is invariant, but for which $\operatorname{coniconv}\left(\left\{\widetilde{v}_{i}\right\}\right)$ is not invariant for $\widetilde{\mathcal{M}}$ is given by the set $\mathcal{M}=\left\{A_{1}, A_{2}\right\}$ with

$$
A_{1}=\left(\begin{array}{rrr}
0 & 1 & 1 \\
1 & 0 & 0 \\
0 & -1 & 0
\end{array}\right), \quad A_{2}=\left(\begin{array}{rrr}
0 & 1 & 0 \\
-1 & 0 & 1 \\
-1 & 0 & 0
\end{array}\right)
$$

The SMP is $P=A_{1} A_{2}$ and has a real leading eigenvector. An extremal real centrally symmetric polytope has been computed by the algorithm described in [17] and gives six vertices:

$$
v_{1}=x, \quad v_{2}=A_{1}^{\prime} v_{1}, \quad v_{3}=A_{2}^{\prime} v_{1}, \quad v_{4}=A_{2}^{\prime} v_{2}, \quad v_{5}=A_{2}^{\prime} v_{3}, \quad v_{6}=A_{1}^{\prime} v_{5},
$$

where $A_{i}^{\prime}=A_{i} / \rho(P), i=1,2$.

The simple algebraic test that $\widetilde{w}=\widetilde{A}_{2}^{\prime} \widetilde{v}_{2}$ does not belong to the conitope shows that computing the set of lifted vertices yields a conitope which is not invariant.

6.2. Extensive tests. In this section we provide an extensive test in order to compare our algorithms to those based on classical polytope norms.

These tests have been done with MATLAB 7.14.0.739 (R2012a) on a two AMD Quad-Core Opteron $23542.2 \mathrm{GHz}$ server with 15.5 GB RAM and 64 bit Red Hat Linux 4.4.6 (kernel 2.6.32).

We used MATLAB function randn to generate real and complex families of two matrices. We scaled each matrix to have spectral radius 1.

For every family we first identified a candidate SMP applying the algorithm presented in [8] and then we produced the unit ball of a polytope extremal norm by means of the algorithm presented in [17] and the unit ball of a conitope extremal norm using Algorithm 1 described in section 3.1.

We organize results by length of the SMP and we consider only lengths corresponding to 20 or more experiences. For each length we plot the 2.5th and 97.5th percentiles (blue (in the electronic version) bars for polytopes and red (in the electronic version) bars for conitopes) and medians (blue (in the electronic version) crosses and red (in the electronic version) stars for polytopes and conitopes, respectively).

In Figure 1 we show results obtained from 12334 tests on randomly generated real families of two matrices $3 \times 3$ with real leading eigenvalue in the SMP. Figure 1(a) 


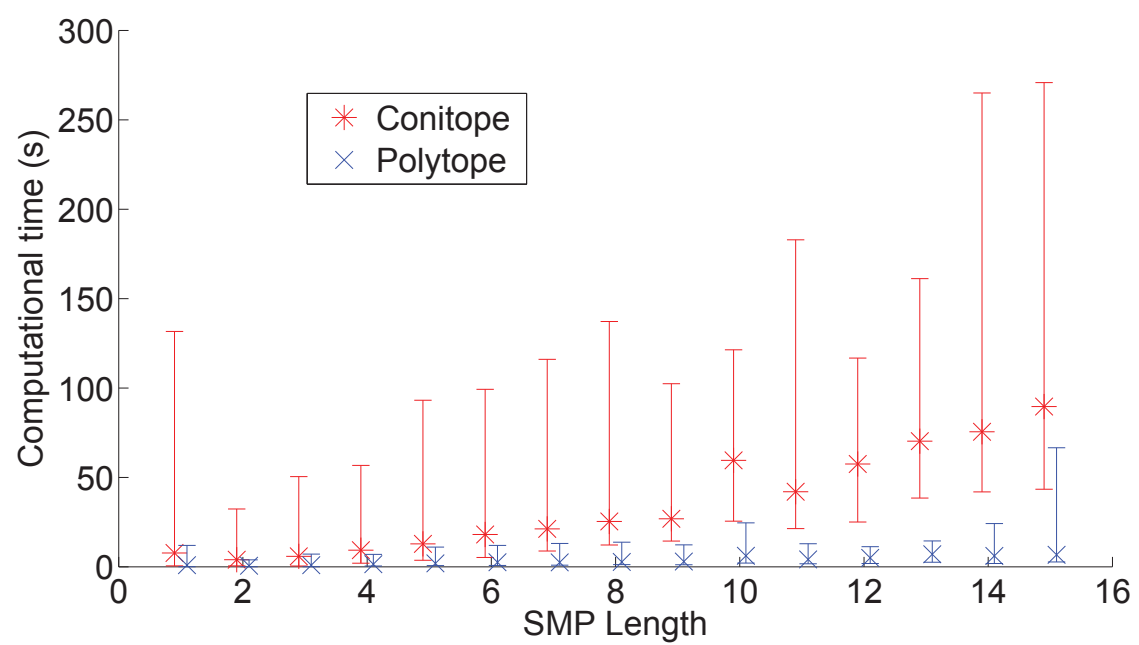

(a)

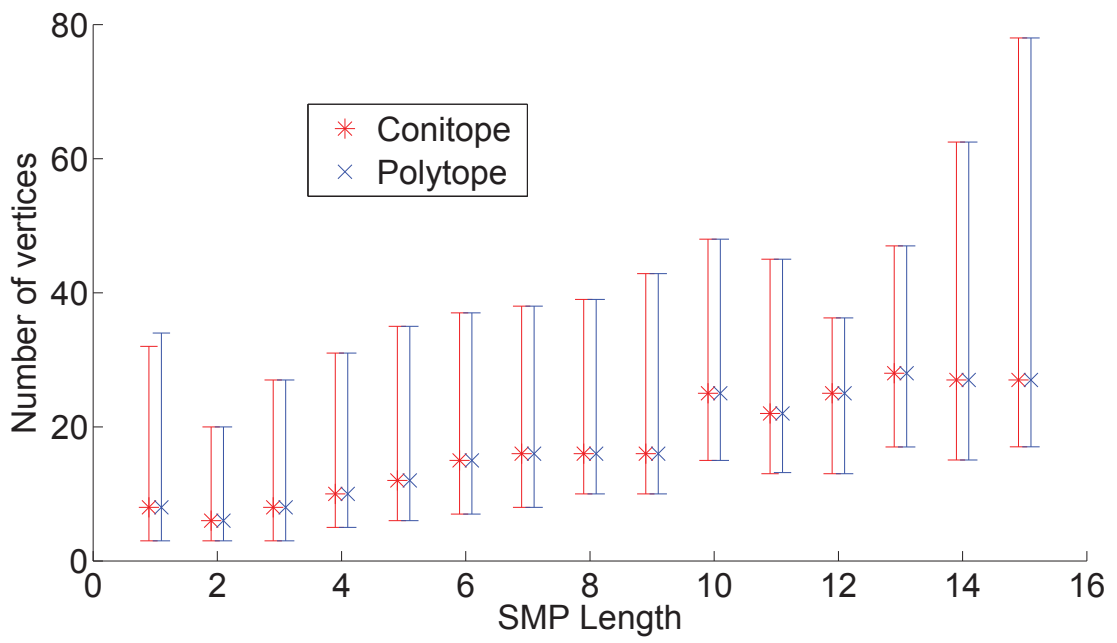

(b)

FIG. 1. Families of two real matrices $3 \times 3$ (12334 families tested). (a) Time for the computation of extremal conitope and polytope norms. (b) Number of vertices in the unit ball of extremal conitope and polytope norms.

contains the computational time required by the polytope method and conitope Algorithm 1 in order to generate the unit ball of an extremal norm. Figure 1(b) reports statistics on the number of vertices of unit balls of extremal polytope and conitope norms. In case of real matrices the two techniques produce unit balls that contain most of the time the same number of vertices; see Figure 1(b). However, the polytope algorithm for real families checks the polytope norm of new vertices through linear programming while the proposed technique uses semidefinite programming to make the same computation. Hence the polytope algorithm performs better than the proposed technique from a computational time point of view in case of families of real 


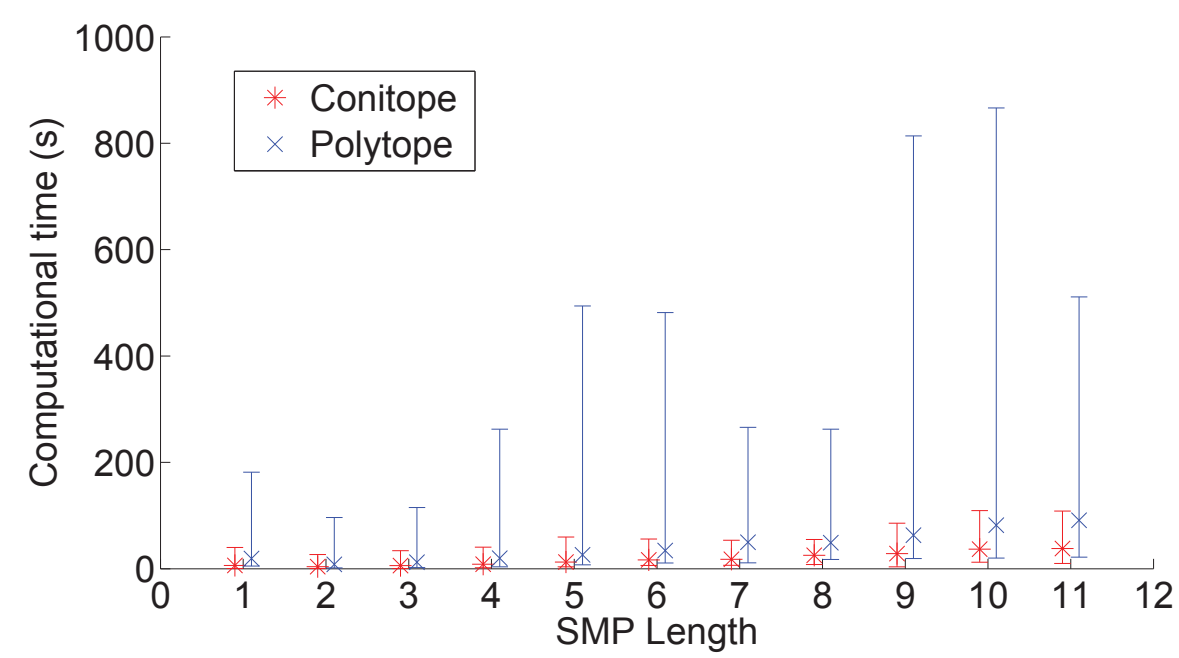

(a)

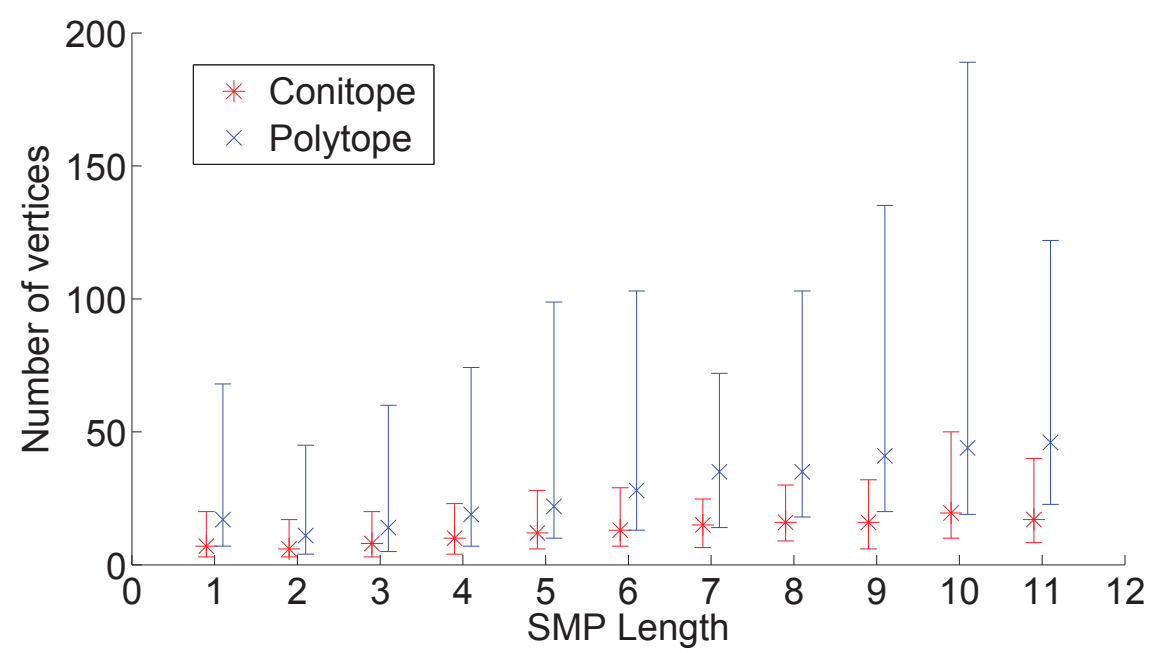

(b)

FIG. 2. Families of two complex matrices $3 \times 3$ ( 8413 families tested). (a) Time for the computation of extremal conitope and polytope norms. (b) Number of vertices in the unit ball of extremal conitope and polytope norms.

matrices with real leading eigenvectors; see Figure 1(a). In fact the mean computational time out of all the 12334 tests is around 17 seconds for conitope Algorithm 1 and 2 seconds for the polytope method.

Figure 2 instead contains the results of 8413 tests on randomly generated complex families of two matrices $3 \times 3$. Figure 2 (a) displays the computational time required by the two techniques to generate extremal norms, Figure 2(b) shows statistics on the number of vertices of unit balls of extremal polytope and conitope norms.

What we conclude is that, in case of complex families of matrices, Algorithm 1 requires less computational time than the polytope algorithm. The mean computational 
time out of all the 8413 tests is around 11 seconds for our conitope Algorithm 1 and 43 seconds for the polytope method. We observe that the number of vertices in the unit ball of an extremal conitope norm is, in general, smaller than the one in the unit ball of an extremal polytope norm; see Figure 2(b). This is related to the partial ordering induced by the invariant cone which allows us to discard products of matrices.

We have also conducted numerical tests on real families of two matrices $3 \times 3$ and $6 \times 6$ with complex and conjugate leading eigenvalues in the SMP, but the number of cases we were able to test is small, the length of the SMP we produced is no more than two, and there is no evidence from these tests that one of the two methods performs better than the other one from a statistical point of view. We finally report that for a randomly generated real family (at least for families of two matrices), it seems that the probability of having a real leading eigenvalue is much higher than the one of a complex conjugate pair. We have no rigorous explanation for this but we think that it could lead to interesting research questions. In the same vein, we observe that complex conjugate cases are often associated to a very small length of the SMP (typically no more than 2).

7. Conclusion. In this paper we introduced a new method for analyzing the asymptotic behavior of semigroups of matrices. By combining the lifting technique and the extremal polytope technique, we have shown that one can, in the case of complex families of matrices, outperform the other algorithms in the literature. We have also provided a sufficient condition for the existence of an extremal norm for the lifted matrices ("an extremal conitope").

A huge advantage of our algorithms is that they allow one to discard in a systematic way many products in the semigroup, thanks to the partial order relation induced by the semidefinite cone. This can greatly reduce the computation time in some situations. Another advantage is that the algorithm can terminate in finite time, and provide a proof that a particular product is actually an SMP, by exhibiting a convex set (the extremal conitope) which is an extremal norm. The same ideas can be applied for the joint spectral subradius, giving potentially a first method for computing the joint spectral subradius of an arbitrary family. We leave this fact for further publication.

The main computational burden, at every step, is in the computation of the quantity $|A v|_{V}$, i.e., the semidefinite program allowing one to compute the conitope norm of the new vertices. We leave it as an open question whether it is possible to apply similar ideas to general matrices with a simpler cone, on which the computations are less expensive.

Acknowledgments. This work was carried out while RJ was visiting the University of L'Aquila. This author wants to thank the university for its outstanding hospitality. The authors thank the anonymous referees for all their insightful comments and constructive remarks. A MATLAB implementation of the methods developed here is available in the JSR Toolbox [34].

\section{REFERENCES}

[1] A. A. Ahmadi, R. M. Jungers, P. A. Parrilo, and M. Roozbehani, Analysis of the joint spectral radius via Lyapunov functions on path-complete graphs, in Hybrid Systems: Computation and Control, Lecture Notes in Comput. Sci. 2011, Springer, Berlin, 2011.

[2] N. E. Barabanov, Lyapunov indicator of discrete inclusions, I-III, Automat. Remote Control, 49 (1988), pp. 152-157, 283-287, 558-565. 
[3] M. A. Berger And Y. WAng, Bounded semigroups of matrices, Linear Algebra Appl., 166 (1992), pp. 21-27.

[4] A. Berman, Cones, Matrices and Mathematical Programming, Lecture Notes in Economics and Mathematical Systems 79, Springer-Verlag, Berlin, 1973.

[5] V. D. Blondel And Y. Nesterov, Computationally efficient approximations of the joint spectral radius, SIAM J. Matrix Anal. Appl., 27 (2005), pp. 256-272.

[6] S. Boyd and L. Vandenberghe, Convex Optimization, Cambridge University Press, Cambridge, UK, 2004.

[7] Y. Chitour, P. Mason, And M. Sigalotti, On the marginal instability of linear switched systems, Systems Control Lett., 61 (2012), pp. 747-757.

[8] A. Cicone and V. Protasov, Fast Computation of Tight Bounds for the Joint Spectral Radius, preprint, MATLAB code available at http://www.mathworks.com/matlabcentral/ fileexchange/36460-joint-spectral-radius-computation, 2013.

[9] J. DaAfouz and J. Bernussou, Parameter dependent Lyapunov functions for discrete time systems with time varying parametric uncertainties, Systems Control Lett., 43 (2001), pp. $355-359$.

[10] I. Daubechies and J. C. Lagarias, Sets of matrices all infinite products of which converge, Linear Algebra Appl., 161 (1992), pp. 227-263.

[11] L. ElSner, The generalized spectral-radius theorem: An analytic-geometric proof, Linear Algebra Appl., 220 (1995), pp. 151-159.

[12] E. Fornasini And M.-E. Valcher, Stability and stabilizability criteria for discrete-time positive switched systems, IEEE Trans. Automat. Control, 57 (2012), pp. 1208-1221,

[13] N. Guglielmi and V. Protasov, Exact computation of joint spectral characteristics of linear operators, Found. Comput. Math., 13 (2013), pp. 37-97.

[14] N. Guglielmi, F. Wirth, and M. Zennaro, Complex polytope extremality results for families of matrices, SIAM J. Matrix Anal. Appl., 27 (2005), pp. 721-743.

[15] N. Guglielmi and M. Zennaro, On the asymptotic properties of a family of matrices, Linear Algebra Appl., 322 (2001), pp. 169-192.

[16] N. Guglielmi and M. Zennaro, Balanced complex polytopes and related vector and matrix norms, J. Convex Anal., 14 (2007), pp. 729-766.

[17] N. Guglielmi and M. Zennaro, An algorithm for finding extremal polytope norms of matrix families, Linear Algebra Appl., 428 (2008), pp. 2265-2282.

[18] N. Guglielmi and M. Zennaro, Finding extremal complex polytope norms for families of real matrices, SIAM J. Matrix Anal. Appl., 31 (2009), pp. 602-620.

[19] R. M. Jungers, The Joint Spectral Radius, Theory and Applications, Lecture Notes in Control and Inform. Sci. 385, Springer-Verlag, Berlin, 2009.

[20] R. M. Jungers, On asymptotic properties of matrix semigroups with an invariant cone, Linear Algebra Appl., 437 (2012), pp. 1205-1214.

[21] R. M. Jungers and V. Y. Protasov, Counterexamples to the complex polytope extremality conjecture, SIAM J. Matrix Anal. Appl., 31 (2009), pp. 404-409.

[22] V. S. KozYAKIn, Algebraic unsolvability of a problem on the absolute stability of desynchronized systems, Automat. Remote Control, 51 (1990), pp. 754-759.

[23] J.-W. Lee and G. E. Dullerud, Uniform stabilization of discrete-time switched and Markovian jump linear systems, Automatica J. IFAC, 42 (2006), pp. 205-218.

[24] D. Liberzon, Switching in Systems and Control, Birkhäuser Boston, Boston, MA, 2003.

[25] T. Monovich and M. Margaliot, Analysis of discrete-time linear switched systems: A variational approach, SIAM J. Control Optim., 49 (2011), pp. 808-829.

[26] I. D. Morris, A new sufficient condition for the uniqueness of Barabanov norms, SIAM J. Matrix Anal. Appl., 33 (2012), pp. 317-324.

[27] P. A. Parrilo and A. Jadbabaie, Approximation of the joint spectral radius using sum of squares, Linear Algebra Appl., 428 (2008), pp. 2385-2402.

[28] V. Yu. Protasov, A generalized joint spectral radius. A geometric approach, Izv. Math., 61 (1997), pp. 995-1030.

[29] V. Yu. Protasov, R. M. Jungers, and V. D. Blondel, Joint spectral characteristics of matrices: A conic programming approach, SIAM J. Matrix Anal. Appl., 31 (2010), pp. $2146-2162$.

[30] M. A. Rami, V. S. Bokharaie, O. Mason, and F. Wirth, Extremal norms for positive linear inclusions, in Proceedings of the 20th International Symposium on Mathematical Theory of Networks and Systems, Melbourne, Australia, 2012.

[31] G. C. Rota And W. G. Strang, A note on the joint spectral radius, Indag. Math., 22 (1960), pp. $379-381$.

[32] R. Shorten, F. Wirth, O. Mason, K. Wulff, and C. King, Stability criteria for switched and hybrid systems, SIAM Rev., 49 (2007), pp. 545-592. 
[33] G. Strang, The joint spectral radius, commentary by Gilbert Strang on paper number 5, in Collected Works of Gian-Carlo Rota, 2001.

[34] G. Vankeerberghen, J. M. Hendrickx, and R. M. Jungers, JSR: A toolbox to compute the joint spectral radius, in Proceedings of Hybrid Systems: Computation and Control (HSCC'14), Berlin, 2014; MATLAB code available at http://www.mathworks.com/ matlabcentral/fileexchange/33202-the-jsr-toolbox.

[35] F. WiRTh, The generalized spectral radius and extremal norms, Linear Algebra Appl., 342 (2002), pp. 17-40. 